\title{
Majorization theorems for strongly convex functions
}

\author{
Syed Zaheer Ullah', Muhammad Adil Khan ${ }^{1}$ and Yu-Ming Chu ${ }^{2 *}$ (B)
}

\section{"Correspondence:}

chuyuming2005@126.com

2 Department of Mathematics, Huzhou University, Huzhou, China

Full list of author information is

available at the end of the article

\begin{abstract}
In the article, we present several majorization theorems for strongly convex functions and give their applications in inequality theory. The given results are the improvement and generalization of the earlier results.
\end{abstract}

MSC: 26D15; 26A51; 39B62

Keywords: Convexity; Strongly convex function; Majorization theorem

\section{Introduction}

Convexity is one the most important, natural, and fundamental concepts in mathematics [1-18]. Convex functions were proposed by Jensen over 100 years ago. Over the past few years, many generalizations and extensions have been made for the convexity, for example, quasi-convexity [19], strong convexity [20, 21], approximate convexity [22], logarithmical convexity [23], midconvexity [24], pseudo-convexity [25], $h$-convexity [26], deltaconvexity [27], $s$-convexity [28], preinvexity [29], GA-convexity [30], GG-convexity [31], coordinate strong convexity [32], and Schur convexity [33-44]. In particular, many remarkable inequalities can be found in the literature [45-79] via the convexity theory.

In the article, we deal with the strongly convex function [20, 21].

Definition 1.1 Let $\Psi$ be a real-valued function defined on an interval $I \subseteq \mathbb{R}$ and $c$ be a positive real number. Then $\Psi$ is said to be strongly convex with modulus $c$ on $I$ if the inequality

$$
\Psi(\eta u+(1-\eta) v) \leq \eta \Psi(u)+(1-\eta) \Psi(v)-c \eta(1-\eta)(u-v)^{2}
$$

holds for all $u, v \in I$ and $\eta \in[0,1]$.

If $\Psi$ is strongly convex, then we clearly see that

$$
\Psi(v)+\Psi_{+}^{\prime}(v)(u-v)+c(u-v)^{2} \leq \Psi(u) .
$$

Next we recall some basic concepts in the theory of majorization.

There are definite natural applications to the indefinite notion that the entries of $n$-tuple $\delta$ are more nearly equal, or less spread out, than the entries of $n$-tuple $\zeta$. The concept arises

(c) The Author(s) 2019. This article is distributed under the terms of the Creative Commons Attribution 4.0 International License (http://creativecommons.org/licenses/by/4.0/), which permits unrestricted use, distribution, and reproduction in any medium, provided you give appropriate credit to the original author(s) and the source, provide a link to the Creative Commons license, and indicate if changes were made. 
in a variety of contexts, and it can be made precise in a number of ways. But in remarkably many cases, the applicable statement is that $\delta$ majorizes $\zeta$ means that the sum of $k$ largest entries of $\zeta$ does not exceed the sum of $k$ largest entries of $\delta$ for all $k=1,2, \ldots, n-1$ with equality for $k=n$. That is, let $\delta=\left(\delta_{1}, \delta_{2}, \ldots, \delta_{n}\right)$ and $\zeta=\left(\zeta_{1}, \zeta_{2}, \ldots, \zeta_{n}\right)$ be two $n$-tuples of real numbers, and

$$
\delta_{1}^{\downarrow} \geq \delta_{2}^{\downarrow} \geq \cdots \geq \delta_{n}^{\downarrow}, \quad \zeta_{1}^{\downarrow} \geq \zeta_{2}^{\downarrow} \geq \cdots \geq \zeta_{n}^{\downarrow}
$$

be their ordered arrangement. Then the $n$-tuple $\delta$ is said to majorize $\zeta$ (or $\zeta$ is to be majorized by $\delta$ ), in symbols we write $\delta \succ \zeta$ if

$$
\sum_{j=1}^{k} \delta_{j}^{\downarrow} \geq \sum_{j=1}^{k} \zeta_{j}^{\downarrow}
$$

holds for $k=1,2, \ldots, n-1$, and

$$
\sum_{j=1}^{n} \delta_{j}=\sum_{j=1}^{n} \zeta_{j} .
$$

Recently, many articles that were published in an extensive variety of fields have been dedicated to the theory of majorization. Undeniably, many concepts of majorization have been reinvented and used in different fields of research such as dominance ordering or Lorenz in economics, graph theory, and optimization. Here we mention useful contexts of majorization in physical science. In physics and chemistry, the terms " $\delta$ is more chaotic than $\zeta$ " " $\delta$ is more mixed than $\zeta$," and " $\delta$ is more disordered than $\zeta$ " are related to inequality ordering and the concept of majorization $\delta \prec \zeta$. To explain "mixing" term, we assume undistinguishable cylindrical beakers which contain liquid of different amounts. Since some beakers contain a large amount of liquid "mixed" to the lesser amounts of liquid from another beaker, such process is known as majorization. The "chaotic" term is used in physical laws. Let us consider one vector which is more chaotic than another vector, it means that one vector majorizes the other vector. The origin of this term is basically related to entropy. In an angulus manner, one vector is said to be more random than another; so, here it means one majorizes the other.

The following distinguished majorization theorem can be found in the literature [80].

Theorem 1.2 Let $\left[\lambda_{1}, \xi_{1}\right] \subseteq \mathbb{R}$ be an interval, and $\delta=\left(\delta_{1}, \delta_{2}, \ldots, \delta_{n}\right)$ and $\zeta=\left(\zeta_{1}, \zeta_{2}, \ldots, \zeta_{n}\right)$ be two n-tuples such that $\delta_{j}, \zeta_{j} \in\left[\lambda_{1}, \xi_{1}\right]$ for $j=1,2, \ldots, n$. Then the inequality

$$
\sum_{j=1}^{n} \Psi\left(\delta_{j}\right) \geq \sum_{j=1}^{n} \Psi\left(\zeta_{j}\right)
$$

holds for every continuous convex function $\Psi:\left[\lambda_{1}, \xi_{1}\right] \rightarrow \mathbb{R}$ if and only if $\boldsymbol{\delta} \succ \zeta$.

A weighted version of Theorem 1.2 was proved by Fuchs [81]. 
Theorem 1.3 Let $\boldsymbol{\delta}=\left(\delta_{1}, \delta_{2}, \ldots, \delta_{n}\right), \boldsymbol{\zeta}=\left(\zeta_{1}, \zeta_{2}, \ldots, \zeta_{n}\right)$ be two decreasing $n$-tuples and $\mathbf{p}=$ $\left(p_{1}, p_{2}, \ldots, p_{n}\right)$ be a real $n$-tuple such that

$$
\begin{aligned}
\sum_{j=1}^{k} p_{j} \delta_{j} & \geq \sum_{j=1}^{k} p_{j} \zeta_{j} \quad \text { for } k=1,2, \ldots, n-1 \text { and } \\
\sum_{j=1}^{n} p_{j} \delta_{j} & =\sum_{j=1}^{n} p_{j} \zeta_{j} .
\end{aligned}
$$

Then, for every continuous convex function $\Psi:\left[\lambda_{1}, \xi_{1}\right] \rightarrow \mathbb{R}$, we have

$$
\sum_{j=1}^{n} p_{j} \Psi\left(\delta_{j}\right) \geq \sum_{j=1}^{n} p_{j} \Psi\left(\zeta_{j}\right)
$$

The following theorem [82] is a weighted majorization theorem for certain $n$-tuples and positive weights.

Theorem 1.4 Let $\Psi:\left[\lambda_{1}, \xi_{1}\right] \rightarrow \mathbb{R}$ be a continuous convex function on an interval $\left[\lambda_{1}, \xi_{1}\right]$, $\mathbf{p}$ be a positive $n$-tuple, and $\delta, \zeta \in\left[\lambda_{1}, \xi_{1}\right]^{n}$ satisfying (1.4) and (1.5).

(a) If the $n$-tuple $\zeta$ is decreasing, then inequality (1.6) holds.

(b) If the $n$-tuple $\boldsymbol{\delta}$ is increasing, then the reverse inequality in (1.6) holds.

Dragomir [83] proved the majorization theorem without using condition (1.4).

Theorem 1.5 Let $\Psi:\left[\lambda_{1}, \xi_{1}\right] \rightarrow \mathbb{R}$ be a continuous convex function on an interval $\left[\lambda_{1}, \xi_{1}\right]$. Suppose that $\delta, \zeta \in\left[\lambda_{1}, \xi_{1}\right]^{n}$ and $p_{j} \geq 0$ for $j=1,2, \ldots, n$. If $\left(\delta_{j}-\zeta_{j}\right)_{(j=\overline{1, n})}$ and $\left(\zeta_{j}\right)_{(j=\overline{1, n})}$ are nondecreasing (nonincreasing) and satisfying (1.5), then inequality (1.6) holds.

The main purpose of the article is to establish the majorization theorem for majorized $n$-tuples by using a strongly convex function and give their applications in the theory of majorization.

\section{Main results}

We start with establishing some inequalities which will be used to prove majorization theorem for strongly convex functions.

Proposition 2.1 Let $\Psi: I \rightarrow \mathbb{R}$ be a strongly convex function with modulus $c$ and $u_{1}, v_{1}, x_{1}, y_{1} \in I$ such that $u_{1}<v_{1} \leq y_{1}<x_{1}$, then the following inequalities hold:
(a) $\frac{\Psi\left(v_{1}\right)-\Psi\left(u_{1}\right)}{v_{1}-u_{1}}-c\left(v_{1}-u_{1}\right) \leq \frac{\Psi\left(x_{1}\right)-\Psi\left(u_{1}\right)}{x_{1}-u_{1}}-c\left(x_{1}-u_{1}\right)$,
(b) $\frac{\Psi\left(v_{1}\right)-\Psi\left(u_{1}\right)}{v_{1}-u_{1}}-c\left(v_{1}-u_{1}\right) \leq \frac{\Psi\left(x_{1}\right)-\Psi\left(v_{1}\right)}{x_{1}-v_{1}}-c\left(x_{1}-v_{1}\right)$,
(c) $\frac{\Psi\left(x_{1}\right)-\Psi\left(u_{1}\right)}{x_{1}-u_{1}}-c\left(x_{1}-u_{1}\right) \leq \frac{\Psi\left(x_{1}\right)-\Psi\left(v_{1}\right)}{x_{1}-v_{1}}-c\left(x_{1}-v_{1}\right)$,
(d) $\frac{\Psi\left(v_{1}\right)-\Psi\left(u_{1}\right)}{v_{1}-u_{1}}-c\left(v_{1}-u_{1}\right) \leq \frac{\Psi\left(x_{1}\right)-\Psi\left(y_{1}\right)}{x_{1}-y_{1}}-c\left(x_{1}-y_{1}\right)$. 
Proof (a) Since $u_{1}<v_{1}<x_{1}$, we obtain

$$
\begin{aligned}
& v_{1}=\left(\frac{x_{1}-v_{1}}{x_{1}-u_{1}}\right) u_{1}+\left(\frac{v_{1}-u_{1}}{x_{1}-u_{1}}\right) x_{1}, \\
& \Psi\left(v_{1}\right)=\Psi\left[\left(\frac{x_{1}-v_{1}}{x_{1}-u_{1}}\right) u_{1}+\left(\frac{v_{1}-u_{1}}{x_{1}-u_{1}}\right) x_{1}\right] .
\end{aligned}
$$

By using strong convexity, we have

$$
\begin{aligned}
& \Psi\left(v_{1}\right) \leq \frac{x_{1}-v_{1}}{x_{1}-u_{1}} \Psi\left(u_{1}\right)+\frac{v_{1}-u_{1}}{x_{1}-u_{1}} \Psi\left(x_{1}\right)-c \frac{x_{1}-v_{1}}{x_{1}-u_{1}} \frac{v_{1}-u_{1}}{x_{1}-u_{1}}\left(u_{1}-x_{1}\right)^{2} \\
& =\left(1-\frac{v_{1}-u_{1}}{x_{1}-u_{1}}\right) \Psi\left(u_{1}\right)+\frac{v_{1}-u_{1}}{x_{1}-u_{1}} \Psi\left(x_{1}\right)-c\left(x_{1}-v_{1}\right)\left(v_{1}-u_{1}\right), \\
& \Psi\left(v_{1}\right)-\Psi\left(u_{1}\right) \leq \frac{v_{1}-u_{1}}{x_{1}-u_{1}} \Psi\left(x_{1}\right)-\frac{v_{1}-u_{1}}{x_{1}-u_{1}} \Psi\left(u_{1}\right)-c\left(x_{1}-v_{1}\right)\left(v_{1}-u_{1}\right), \\
& \frac{\Psi\left(v_{1}\right)-\Psi\left(u_{1}\right)}{v_{1}-u_{1}} \leq \frac{\Psi\left(x_{1}\right)-\Psi\left(u_{1}\right)}{x_{1}-u_{1}}-c\left(x_{1}-v_{1}\right), \\
& \frac{\Psi\left(v_{1}\right)-\Psi\left(u_{1}\right)}{v_{1}-u_{1}} \leq \frac{\Psi\left(x_{1}\right)-\Psi\left(u_{1}\right)}{x_{1}-u_{1}}-c\left(1-\frac{v_{1}-u_{1}}{x_{1}-u_{1}}\right)\left(x_{1}-u_{1}\right) .
\end{aligned}
$$

Rearranging the above inequalities, we deduce (a).

(b) From (a) we can write

$$
\begin{aligned}
& \Psi\left(v_{1}\right)-\Psi\left(u_{1}\right)-c\left(v_{1}-u_{1}\right)^{2} \\
& \leq\left(\frac{v_{1}-u_{1}}{x_{1}-u_{1}}\right)\left\{\Psi\left(x_{1}\right)-\Psi\left(u_{1}\right)-c\left(x_{1}-u_{1}\right)^{2}\right\} \\
& =\left(\frac{v_{1}-u_{1}}{x_{1}-u_{1}}\right)\left\{\Psi\left(x_{1}\right)-\Psi\left(v_{1}\right)+\Psi\left(v_{1}\right)-\Psi\left(u_{1}\right)-c\left(x_{1}-u_{1}\right)^{2}\right\} \text {, } \\
& \left\{\Psi\left(v_{1}\right)-\Psi\left(u_{1}\right)\right\}\left(1-\frac{v_{1}-u_{1}}{x_{1}-u_{1}}\right)-c\left(v_{1}-u_{1}\right)^{2} \\
& \leq\left(\frac{v_{1}-u_{1}}{x_{1}-u_{1}}\right)\left\{\Psi\left(x_{1}\right)-\Psi\left(v_{1}\right)-c\left(x_{1}-u_{1}\right)^{2}\right\}, \\
& \left\{\Psi\left(v_{1}\right)-\Psi\left(u_{1}\right)\right\}\left(x_{1}-v_{1}\right)-c\left(v_{1}-u_{1}\right)^{2}\left(x_{1}-u_{1}\right) \\
& \leq\left(v_{1}-u_{1}\right)\left\{\Psi\left(x_{1}\right)-\Psi\left(v_{1}\right)-c\left(x_{1}-u_{1}\right)^{2}\right\}, \\
& \left(x_{1}-v_{1}\right)\left[\Psi\left(v_{1}\right)-\Psi\left(u_{1}\right)-c\left(v_{1}-u_{1}\right)^{2}\left(1-\frac{u_{1}-v_{1}}{x_{1}-v_{1}}\right)\right] \\
& \leq\left(v_{1}-u_{1}\right)\left\{\Psi\left(x_{1}\right)-\Psi\left(v_{1}\right)-c\left(x_{1}-u_{1}\right)^{2}\right\}, \\
& \left(x_{1}-v_{1}\right)\left[\Psi\left(v_{1}\right)-\Psi\left(u_{1}\right)-c\left(v_{1}-u_{1}\right)^{2}\right] \\
& \leq\left(v_{1}-u_{1}\right)\left\{\Psi\left(x_{1}\right)-\Psi\left(v_{1}\right)\right\}-c\left(v_{1}-u_{1}\right)\left[\left(x_{1}-u_{1}\right)^{2}-\left(v_{1}-u_{1}\right)^{2}\right] \\
& =\left(v_{1}-u_{1}\right)\left[\left(\Psi\left(x_{1}\right)-\Psi\left(v_{1}\right)\right)-c\left(x_{1}-v_{1}\right)\left(x_{1}-2 u_{1}+v_{1}\right)\right] \\
& \leq\left(v_{1}-u_{1}\right)\left[\left(\Psi\left(x_{1}\right)-\Psi\left(v_{1}\right)\right)-c\left(x_{1}-v_{1}\right)^{2}\right] \text {. }
\end{aligned}
$$

Rearranging the above inequalities, we deduce (b). 
(c) Now, we can write

$$
\begin{aligned}
& \frac{\Psi\left(x_{1}\right)-\Psi\left(u_{1}\right)-c\left(x_{1}-u_{1}\right)^{2}}{x_{1}-u_{1}} \\
& \quad=\frac{\left\{\Psi\left(x_{1}\right)-\Psi\left(v_{1}\right)\right\}\left(x_{1}-v_{1}\right)}{\left(x_{1}-u_{1}\right)\left(x_{1}-v_{1}\right)}+\frac{\left\{\Psi\left(v_{1}\right)-\Psi\left(u_{1}\right)\right\}\left(v_{1}-u_{1}\right)}{\left(x_{1}-u_{1}\right)\left(v_{1}-u_{1}\right)}-c\left(x_{1}-u_{1}\right) .
\end{aligned}
$$

Using inequality (a), we obtain

$$
\begin{aligned}
\frac{\Psi\left(x_{1}\right)-\Psi\left(u_{1}\right)-c\left(x_{1}-u_{1}\right)^{2}}{x_{1}-u_{1}} & \frac{x_{1}-v_{1}}{x_{1}-u_{1}}\left(\frac{\Psi\left(x_{1}\right)-\Psi\left(v_{1}\right)}{x_{1}-v_{1}}\right)+\left(\frac{\Psi\left(x_{1}\right)-\Psi\left(u_{1}\right)}{x_{1}-u_{1}}-c\left(x_{1}-u_{1}\right)+c\left(v_{1}-u_{1}\right)\right) \\
& \times\left(\frac{v_{1}-u_{1}}{x_{1}-u_{1}}\right)-c\left(x_{1}-u_{1}\right), \\
\hline\left(x_{1}\right)-\Psi\left(u_{1}\right)-c\left(x_{1}-u_{1}\right)^{2} & \left.\frac{x_{1}-v_{1}}{x_{1}-u_{1}}\right) \\
\leq & \frac{x_{1}-v_{1}}{x_{1}-u_{1}}\left(\frac{\Psi\left(x_{1}\right)-\Psi\left(v_{1}\right)}{x_{1}-v_{1}}\right)-c\left(x_{1}-u_{1}\right)\left(1-\frac{\left(v_{1}-u_{1}\right)^{2}}{\left(x_{1}-u_{1}\right)^{2}}\right) \\
= & \frac{x_{1}-v_{1}}{x_{1}-u_{1}}\left(\frac{\Psi\left(x_{1}\right)-\Psi\left(v_{1}\right)}{x_{1}-v_{1}}\right)-\frac{c}{\left(x_{1}-u_{1}\right)}\left\{\left(x_{1}-u_{1}\right)^{2}-\left(v_{1}-u_{1}\right)^{2}\right\} \\
= & \frac{x_{1}-v_{1}}{x_{1}-u_{1}}\left(\frac{\Psi\left(x_{1}\right)-\Psi\left(v_{1}\right)}{x_{1}-v_{1}}\right)-\frac{c\left(x_{1}-v_{1}\right)}{\left(x_{1}-u_{1}\right)}\left\{\left(x_{1}-2 u_{1}+v_{1}\right)\right\} \\
\leq & \frac{x_{1}-v_{1}}{x_{1}-u_{1}}\left(\frac{\Psi\left(x_{1}\right)-\Psi\left(v_{1}\right)}{x_{1}-v_{1}}\right)-\frac{c\left(x_{1}-v_{1}\right)}{\left(x_{1}-u_{1}\right)}\left(x_{1}-v_{1}\right) \\
\leq & \frac{\Psi\left(x_{1}\right)-\Psi\left(v_{1}\right)-c\left(x_{1}-v_{1}\right)^{2}}{x_{1}-v_{1}}\left(\frac{x_{1}-v_{1}}{x_{1}-u_{1}}\right) .
\end{aligned}
$$

Rearranging the above inequality, we deduce (c).

(d) Similarly, to prove the last inequality using inequality (b) and if $u_{1}<v_{1}<y_{1}$, then we obtain

$$
\frac{\Psi\left(v_{1}\right)-\Psi\left(u_{1}\right)}{v_{1}-u_{1}}-c\left(v_{1}-u_{1}\right) \leq \frac{\Psi\left(y_{1}\right)-\Psi\left(v_{1}\right)}{y_{1}-v_{1}}-c\left(y_{1}-v_{1}\right)
$$

also, if $v_{1}<y_{1}<x_{1}$, then we obtain

$$
\frac{\Psi\left(y_{1}\right)-\Psi\left(v_{1}\right)}{y_{1}-v_{1}}-c\left(y_{1}-v_{1}\right) \leq \frac{\Psi\left(x_{1}\right)-\Psi\left(y_{1}\right)}{x_{1}-y_{1}}-c\left(x_{1}-y_{1}\right)
$$

from (2.1) and (2.2), we deduce (d).

Remark 2.2 If $\Psi$ is a strongly convex function with modulus $c$, then, by using inequalities in Proposition 2.1, we clearly see that the function $\hbar(x, y)=\frac{\Psi(x)-\Psi(y)-c(x-y)^{2}}{x-y}$ is increasing in both variables.

Now we are in the position to prove majorization theorem for strongly convex functions. 
Theorem 2.3 Let $\Psi:\left[\lambda_{1}, \xi_{1}\right] \rightarrow \mathbb{R}$ be a strongly convex function with modulus c. Suppose that $\delta=\left(\delta_{1}, \delta_{2}, \ldots, \delta_{n}\right)$ and $\zeta=\left(\zeta_{1}, \zeta_{2}, \ldots, \zeta_{n}\right)$ are $n$-tuples, $\delta_{j}, \zeta_{j} \in\left[\lambda_{1}, \xi_{1}\right], j=1,2, \ldots, n$, and the $n$-tuple $\delta$ majorizes $\zeta$. Then the following inequality holds:

$$
\sum_{j=1}^{n} \Psi\left(\delta_{j}\right) \geq \sum_{j=1}^{n} \Psi\left(\zeta_{j}\right)+c \sum_{j=1}^{n}\left(\delta_{j}-\zeta_{j}\right)^{2}
$$

Proof Without loss of generality, we consider that

$$
\delta_{1} \geq \delta_{2} \geq \cdots \geq \delta_{n}, \quad \zeta_{1} \geq \zeta_{2} \geq \cdots \geq \zeta_{n} \quad \text { and } \quad \delta_{j} \neq \zeta_{j} \quad \text { for } j=1,2, \ldots, n
$$

Assume that $d_{j}=\hbar\left(\delta_{j}, \zeta_{j}\right)=\frac{\Psi\left(\delta_{j}\right)-\Psi\left(\zeta_{j}\right)-c\left(\delta_{j}-\zeta_{j}\right)^{2}}{\delta_{j}-\zeta_{j}}$. Since $\Psi$ is strongly convex, so by Remark 2.2, it implies that the sequence $\left\{d_{j}\right\}_{j=1}^{n}$ is decreasing. Suppose that

$$
E_{j}=\sum_{k=1}^{j} \delta_{k} \quad \text { and } \quad F_{j}=\sum_{k=1}^{j} \zeta_{k}, \quad j=1,2, \ldots, n, E_{0}=F_{0}=0
$$

Since $\delta \succ \zeta$, therefore

$$
E_{n}=F_{n} \quad \text { and } \quad E_{j} \geq F_{j}, \quad \text { for } j=1,2, \ldots, n-1 .
$$

Now, we can write

$$
\begin{gathered}
\sum_{j=1}^{n} \Psi\left(\delta_{j}\right)-\sum_{j=1}^{n} \Psi\left(\zeta_{j}\right)=\sum_{j=1}^{n}\left\{\Psi\left(\delta_{j}\right)-\Psi\left(\zeta_{j}\right)-c\left(\delta_{j}-\zeta_{j}\right)^{2}+c\left(\delta_{j}-\zeta_{j}\right)^{2}\right\} \\
=\sum_{j=1}^{n}\left[\left\{\frac{\Psi\left(\delta_{j}\right)-\Psi\left(\zeta_{j}\right)-c\left(\delta_{j}-\zeta_{j}\right)^{2}}{\delta_{j}-\zeta_{j}}\right\}\left(\delta_{j}-\zeta_{j}\right)+c\left(\delta_{j}-\zeta_{j}\right)^{2}\right],
\end{gathered}
$$

which can be written as

$$
\sum_{j=1}^{n} \Psi\left(\delta_{j}\right)-\sum_{j=1}^{n} \Psi\left(\zeta_{j}\right)-c \sum_{j=1}^{n}\left(\delta_{j}-\zeta_{j}\right)^{2}=\sum_{j=1}^{n} d_{j}\left(\delta_{j}-\zeta_{j}\right)
$$

Clearly,

$$
E_{j}-E_{j-1}=\delta_{j} \quad \text { and } \quad F_{j}-F_{j-1}=\zeta_{j} \quad \text { for } j=1,2, \ldots, n \text {. }
$$

Using (2.5) in (2.4), we get

$$
\begin{aligned}
& \sum_{j=1}^{n} \Psi\left(\delta_{j}\right)-\sum_{j=1}^{n} \Psi\left(\zeta_{j}\right)-c \sum_{j=1}^{n}\left(\delta_{j}-\zeta_{j}\right)^{2} \\
& =\sum_{j=1}^{n} d_{j}\left\{\left(E_{j}-E_{j-1}\right)-\left(F_{j}-F_{j-1}\right)\right\} \\
& =\sum_{j=1}^{n} d_{j}\left(E_{j}-F_{j}\right)-\sum_{j=1}^{n} d_{j}\left(E_{j-1}-F_{j-1}\right)
\end{aligned}
$$




$$
\begin{aligned}
& =\sum_{j=1}^{n} d_{j}\left(E_{j}-F_{j}\right)-\sum_{j=0}^{n-1} d_{j+1}\left(E_{j}-F_{j}\right) \\
& =\sum_{j=1}^{n-1} d_{j}\left(E_{j}-F_{j}\right)-\sum_{j=1}^{n-1} d_{j+1}\left(E_{j}-F_{j}\right) \\
& =\sum_{j=1}^{n-1}\left(d_{j}-d_{j+1}\right)\left(E_{j}-F_{j}\right) .
\end{aligned}
$$

Since $d_{j} \geq d_{j+1}$ and $E_{j} \geq F_{j}$, so $\sum_{j=1}^{n-1}\left(d_{j}-d_{j+1}\right)\left(E_{j}-F_{j}\right) \geq 0$, hence from (2.6) we obtain (2.3).

Example 2.4 Let $\lambda_{1}, \xi_{1} \in \mathbb{R}^{+}$with $\lambda_{1}<\xi_{1}$ and $\alpha, \kappa, \gamma \in\left[\lambda_{1}, \xi_{1}\right]$ such that $2 \alpha, 2 \kappa, 2 \gamma, \alpha+$ $\kappa, \kappa+\gamma, \gamma+\alpha \in\left[\lambda_{1}, \xi_{1}\right]$. Then, for any $c \leq \frac{1}{\xi_{1}^{3}}$, we have the inequality

$$
\frac{1}{2 \alpha}+\frac{1}{2 \kappa}+\frac{1}{2 \gamma} \geq \frac{1}{\alpha+\kappa}+\frac{1}{\kappa+\gamma}+\frac{1}{\gamma+\alpha}+c\left\{(\alpha-\kappa)^{2}+(\kappa-\gamma)^{2}+(\gamma-\alpha)^{2}\right\} .
$$

Solution 2.5 Let $\boldsymbol{\delta}=(2 \alpha, 2 \kappa, 2 \gamma), \zeta=(\alpha+\kappa, \kappa+\gamma, \gamma+\alpha)$, clearly $\boldsymbol{\delta} \succ \boldsymbol{\zeta}$ (for details see [84]). Since the function $\Psi(x)=\frac{1}{x}, x \in\left[\lambda_{1}, \xi_{1}\right]$ is strongly convex with modulus $c \leq \frac{1}{\xi_{1}^{3}}$. Therefore using inequality (2.3) we obtain (2.7).

Example 2.6 Let $\lambda_{1}, \xi_{1} \in \mathbb{R}^{+}$with $\lambda_{1}<\xi_{1}$ and $\alpha, \kappa, \gamma \in\left[\lambda_{1}, \xi_{1}\right]$ such that $\alpha+\kappa-\gamma, \kappa+\gamma-$ $\alpha, \gamma+\alpha-\kappa, \alpha \kappa \gamma \in\left[\lambda_{1}, \xi_{1}\right]$. Then, for any $c \leq \frac{1}{2 \xi_{1}^{2}}$, we have the inequality

$$
\begin{aligned}
& (\alpha+\kappa-\gamma)(\kappa+\gamma-\alpha)(\gamma+\alpha-\kappa) \\
& \quad \leq \alpha \kappa \gamma+\exp \left[-c\left\{(\kappa-\gamma)^{2}+(\gamma-\alpha)^{2}+(\alpha-\kappa)^{2}\right\}\right] .
\end{aligned}
$$

Solution 2.7 Let $\boldsymbol{\delta}=(\alpha+\kappa-\gamma, \kappa+\gamma-\alpha, \gamma+\alpha-\kappa), \boldsymbol{\zeta}=(\alpha, \kappa, \gamma)$, clearly $\boldsymbol{\delta} \succ \boldsymbol{\zeta}$. As $\Psi(x)=\log x, x \in\left[\lambda_{1}, \xi_{1}\right]$ is a strongly concave function with modulus $c \leq \frac{1}{2 \xi_{1}^{2}}$. Therefore using inequality (2.3) we obtain (2.8).

Example 2.8 Let $\left[-\frac{\pi}{6}, \frac{\pi}{6}\right]$ be an interval and $\alpha_{j} \in\left[-\frac{\pi}{6}, \frac{\pi}{6}\right]$ such that $2 \alpha_{j}-\alpha_{j+1} \in\left[-\frac{\pi}{6}, \frac{\pi}{6}\right]$ for $j=1,2, \ldots, n$, with $\alpha_{n+1}=\alpha_{1}$. Then, for any $c \leq \frac{\sqrt{3}}{4}$, one has

$$
\begin{aligned}
& \cos \left(2 \alpha_{1}-\alpha_{2}\right)+\cos \left(2 \alpha_{2}-\alpha_{3}\right)+\cdots+\cos \left(2 \alpha_{n}-\alpha_{1}\right) \\
& \quad \leq \cos \alpha_{1}+\cos \alpha_{2}+\cdots+\cos \alpha_{n}-c\left\{\left(\alpha_{1}-\alpha_{2}\right)^{2}+\cdots+\left(\alpha_{n}-\alpha_{1}\right)^{2}\right\} .
\end{aligned}
$$

Solution 2.9 Let $\boldsymbol{\delta}=\left(2 \alpha_{1}-\alpha_{2}, 2 \alpha_{2}-\alpha_{3}, 2 \alpha_{n}-\alpha_{1}\right), \boldsymbol{\zeta}=\left(\alpha_{1}, \alpha_{2}, \ldots, \alpha_{n}\right)$, clearly $\boldsymbol{\delta} \succ \boldsymbol{\zeta}$ (for details see [84]). Since the function $\Psi(x)=\cos x, x \in\left[-\frac{\pi}{6}, \frac{\pi}{6}\right]$ is strongly concave with modulus $c \leq \frac{\sqrt{3}}{4}$. Therefore using inequality (2.3) we obtain (2.9).

Remark 2.10 We clearly see that inequalities (2.7), (2.8), and (2.9) are the improvements of the corresponding inequalities given in [84].

The weighted version of Theorem 2.3 is given in the following theorem. It can be viewed as a generalization of the majorization theorem. 
Theorem 2.11 Let $\boldsymbol{\delta}=\left(\delta_{1}, \delta_{2}, \ldots, \delta_{n}\right), \zeta=\left(\zeta_{1}, \zeta_{2}, \ldots, \zeta_{n}\right)$ be two decreasing $n$-tuples and $\mathbf{p}=$ $\left(p_{1}, p_{2}, \ldots, p_{n}\right)$ be a real $n$-tuple such that

$$
\begin{aligned}
\sum_{j=1}^{k} p_{j} \delta_{j} & \geq \sum_{j=1}^{k} p_{j} \zeta_{j} \quad \text { for } k=1,2, \ldots, n-1, \quad \text { and } \\
\sum_{j=1}^{n} p_{j} \delta_{j} & =\sum_{j=1}^{n} p_{j} \zeta_{j} .
\end{aligned}
$$

Then, for every strongly convex function $\Psi:\left[\lambda_{1}, \xi_{1}\right] \rightarrow \mathbb{R}$, we have

$$
\sum_{j=1}^{n} p_{j} \Psi\left(\delta_{j}\right) \geq \sum_{j=1}^{n} p_{j} \Psi\left(\zeta_{j}\right)+c \sum_{j=1}^{n} p_{j}\left(\delta_{j}-\zeta_{j}\right)^{2}
$$

Proof The idea of the proof is similar to the idea of the proof of Theorem 2.3.

In the following theorem we shall establish a more general inequality for strongly convex functions.

Theorem 2.12 Let $\Psi:\left[\lambda_{1}, \xi_{1}\right] \rightarrow \mathbb{R}$ be a strongly convex function with modulus $c$. Suppose that $\boldsymbol{\delta}=\left(\delta_{1}, \delta_{2}, \ldots, \delta_{n}\right), \zeta=\left(\zeta_{1}, \zeta_{2}, \ldots, \zeta_{n}\right)$ and $\mathbf{p}=\left(p_{1}, p_{2}, \ldots, p_{n}\right)$ are $n$-tuples such that $\delta_{i}, \zeta_{i} \in\left[\lambda_{1}, \xi_{1}\right], p_{i} \geq 0, j=1,2, \ldots, n$. Then the following inequality holds:

$$
\sum_{j=1}^{n} p_{j} \Psi\left(\delta_{j}\right) \geq \sum_{j=1}^{n} p_{j} \Psi\left(\zeta_{j}\right)+\sum_{j=1}^{n} p_{j} \Psi_{+}^{\prime}\left(\zeta_{j}\right)\left(\delta_{j}-\zeta_{j}\right)+c \sum_{j=1}^{n} p_{j}\left(\delta_{j}-\zeta_{j}\right)^{2} .
$$

Proof Since $\Psi$ is a strongly convex function, therefore we have

$$
\Psi(\delta) \geq \Psi(\zeta)+\Psi_{+}^{\prime}(\zeta)(\delta-\zeta)+c(\delta-\zeta)^{2}
$$

Let $\zeta \rightarrow \zeta_{j}$ and $\delta \rightarrow \delta_{j}$, for $j=1,2, \ldots, n$ in (2.14), we get

$$
\Psi\left(\delta_{j}\right) \geq \Psi\left(\zeta_{j}\right)+\Psi_{+}^{\prime}\left(\zeta_{j}\right)\left(\delta_{j}-\zeta_{j}\right)+c\left(\delta_{j}-\zeta_{j}\right)^{2}
$$

Multiplying (2.15) by $p_{j} \geq 0$ and summing over $j=1,2, \ldots, n$, we get

$$
\sum_{j=1}^{n} p_{j} \Psi\left(\delta_{j}\right) \geq \sum_{j=1}^{n} p_{j} \Psi\left(\zeta_{j}\right)+\sum_{j=1}^{n} p_{j} \Psi_{+}^{\prime}\left(\zeta_{j}\right)\left(\delta_{j}-\zeta_{j}\right)+c \sum_{j=1}^{n} p_{j}\left(\delta_{j}-\zeta_{j}\right)^{2}
$$

Remark 2.13 By setting $\zeta_{j}=\bar{\delta}=\sum_{j=1}^{n} p_{j} \delta_{j}$ for $j=1,2, \ldots, n$ in (2.13), we obtain Jensen's inequality for strongly convex functions which has already been proved by Merentes and Nikodem in [20]. Moreover, by rearranging (2.13) and replacing $\zeta_{j}$ by $\delta_{j}$ and setting $\delta_{j}=\overline{\bar{\Delta}}=\frac{\sum_{j=1}^{n} p_{j} \delta_{j} \Psi_{+}^{\prime}\left(\delta_{j}\right)}{\sum_{j=1}^{n} p_{j} \Psi_{+}^{\prime}\left(\delta_{j}\right)} \in\left[\lambda_{1}, \xi_{1}\right]$ for $j=1,2, \ldots, n$, where $\sum_{j=1}^{n} p_{j} \Psi_{+}^{\prime}\left(\delta_{j}\right) \neq 0$, we obtain Slater's inequality for strongly convex functions.

Next, we prove majorization theorem for positive weight and monotonic condition on a single tuple. 
Theorem 2.14 Let $\Psi:\left[\lambda_{1}, \xi_{1}\right] \rightarrow \mathbb{R}$ be a strongly convex function with modulus $c$. Suppose that $\boldsymbol{\delta}=\left(\delta_{1}, \delta_{2}, \ldots, \delta_{n}\right), \zeta=\left(\zeta_{1}, \zeta_{2}, \ldots, \zeta_{n}\right)$ and $\mathbf{p}=\left(p_{1}, p_{2}, \ldots, p_{n}\right)$ are $n$-tuples such that $\delta_{j}, \zeta_{j} \in\left[\lambda_{1}, \xi_{1}\right], p_{j} \geq 0$ for $j=1,2, \ldots, n$ and they satisfy

$$
\begin{aligned}
\sum_{j=1}^{k} p_{j} \delta_{j} & \geq \sum_{j=1}^{k} p_{j} \zeta_{j} \quad \text { for } k=1,2, \ldots, n-1 \text { and } \\
\sum_{j=1}^{n} p_{j} \delta_{j} & =\sum_{j=1}^{n} p_{j} \zeta_{j} .
\end{aligned}
$$

(a) If the $n$-tuple $\zeta$ is decreasing, then the following inequality holds:

$$
\sum_{j=1}^{n} p_{j} \Psi\left(\delta_{j}\right) \geq \sum_{j=1}^{n} p_{j} \Psi\left(\zeta_{j}\right)+c \sum_{j=1}^{n} p_{j}\left(\delta_{j}-\zeta_{j}\right)^{2} .
$$

(b) If the $n$-tuple $\boldsymbol{\delta}$ is increasing, then the following inequality holds:

$$
\sum_{j=1}^{n} p_{j} \Psi\left(\zeta_{j}\right) \geq \sum_{j=1}^{n} p_{j} \Psi\left(\delta_{j}\right)+c \sum_{j=1}^{n} p_{j}\left(\zeta_{j}-\delta_{j}\right)^{2} .
$$

Proof (a) By using (2.13) and if $\zeta$ is a decreasing $n$-tuple, then we use (2.17) and (2.18), and since $\Psi$ is strongly convex, so $\Psi$ is convex. Therefore using the idea of [82, p. 32], we have $\sum_{j=1}^{n} p_{j} \Psi_{+}^{\prime}\left(\zeta_{j}\right)\left(\delta_{j}-\zeta_{j}\right) \geq 0$. Hence $(2.13)$ can be written as

$$
\sum_{j=1}^{n} p_{j} \Psi\left(\delta_{j}\right)-\sum_{j=1}^{n} p_{j} \Psi\left(\zeta_{j}\right)-c \sum_{j=1}^{n} p_{j}\left(\delta_{j}-\zeta_{j}\right)^{2} \geq \sum_{j=1}^{n} p_{j} \Psi_{+}^{\prime}\left(\zeta_{j}\right)\left(\delta_{j}-\zeta_{j}\right) \geq 0 .
$$

From (2.21), we deduce (2.19).

Similarly we can prove part (b).

The following theorem is in fact the generalization of Theorem 1.5 for a strongly convex function.

Theorem 2.15 Let $\Psi:\left[\lambda_{1}, \xi_{1}\right] \rightarrow \mathbb{R}$ be a strongly convex function with modulus $c$. Suppose that $\boldsymbol{\delta}=\left(\delta_{1}, \delta_{2}, \ldots, \delta_{n}\right), \boldsymbol{\zeta}=\left(\zeta_{1}, \zeta_{2}, \ldots, \zeta_{n}\right)$ and $\mathbf{p}=\left(p_{1}, p_{2}, \ldots, p_{n}\right)$ are $n$-tuples such that $\delta_{j}, \zeta_{j} \in\left[\lambda_{1}, \xi_{1}\right], p_{j} \geq 0$ for $j=1,2, \ldots, n$. If $\left(\delta_{j}-\zeta_{j}\right)_{(j=1, n)}$ and $\left(\zeta_{j}\right)_{(j=1, n)}$ are decreasing (increasing) $n$-tuples and satisfy (2.18), then the following inequality holds:

$$
\sum_{j=1}^{n} p_{j} \Psi\left(\delta_{j}\right) \geq \sum_{j=1}^{n} p_{j} \Psi\left(\zeta_{j}\right)+c \sum_{j=1}^{n} p_{j}\left(\delta_{j}-\zeta_{j}\right)^{2}
$$

Proof The idea of the proof is similar to the idea of the proof of Theorem 2.14.

Remark 2.16 Let all the assumptions of Theorem 2.15 hold. Furthermore, if $\Psi$ is increasing and satisfying (2.17), then (2.22) holds. 
The following theorem is in fact the generalization of [85, Theorem 1] for a strongly convex function.

Theorem 2.17 Let $\theta$ be a strictly increasing function from $\left(\lambda_{1}, \xi_{1}\right)$ onto $\left(\lambda_{2}, \xi_{2}\right), \Psi^{\circ} \theta^{-1}$ be a strongly convex function on $\left[\lambda_{2}, \xi_{2}\right]$, and $\delta=\left(\delta_{1}, \delta_{2}, \ldots, \delta_{n}\right)$ and $\zeta=\left(\zeta_{1}, \zeta_{2}, \ldots, \zeta_{n}\right)$ be n-tuples such that $\delta_{j}, \zeta_{j} \in\left(\lambda_{1}, \xi_{1}\right)(j=1,2, \ldots, n)$ and they satisfy

$$
\begin{aligned}
& \sum_{j=1}^{k} p_{i} \theta\left(\delta_{j}\right) \geq \sum_{j=1}^{k} p_{j} \theta\left(\zeta_{j}\right) \quad \text { for } k=1,2, \ldots, n-1 \text { and } \\
& \sum_{j=1}^{n} p_{j} \theta\left(\delta_{j}\right)=\sum_{j=1}^{n} p_{j} \theta\left(\zeta_{j}\right) .
\end{aligned}
$$

\section{Then the following statements are true:}

(a) If the $n$-tuple $\zeta$ is decreasing and $\Psi$ is a decreasing function, then

$$
\sum_{j=1}^{n} p_{j} \Psi\left(\delta_{j}\right) \geq \sum_{j=1}^{n} p_{j} \Psi\left(\zeta_{j}\right)+c \sum_{j=1}^{n} p_{j}\left(\theta\left(\delta_{j}\right)-\theta\left(\zeta_{j}\right)\right)^{2}
$$

(b) If the $n$-tuple $\delta$ is increasing and $\Psi$ is an increasing function, then

$$
\sum_{j=1}^{n} p_{j} \Psi\left(\zeta_{j}\right) \geq \sum_{j=1}^{n} p_{j} \Psi\left(\delta_{j}\right)+c \sum_{j=1}^{n} p_{j}\left(\theta\left(\delta_{j}\right)-\theta\left(\zeta_{j}\right)\right)^{2}
$$

Proof (a) Let $\theta\left(\delta_{j}\right)=a_{j}, \theta\left(\zeta_{j}\right)=b_{j}, \Psi^{\circ} \theta^{-1}\left(a_{j}\right)=\bar{\Psi}\left(a_{j}\right)$, then by using (1.2), we have

$$
\sum_{j=1}^{k} p_{j} \bar{\Psi}\left(a_{j}\right)-\sum_{j=1}^{k} p_{j} \bar{\Psi}\left(b_{j}\right)+c \sum_{j=1}^{k} p_{j}\left(a_{j}-b_{j}\right)^{2} \geq \sum_{j=1}^{k} p_{j} \bar{\Psi}_{+}^{\prime}\left(b_{j}\right)\left(a_{j}-b_{j}\right) .
$$

Since $\Psi$ is decreasing, by using (2.23) and (2.24) and the idea of Theorem 2.3, we have $\sum_{j=1}^{k} p_{j} \bar{\Psi}_{+}^{\prime}\left(b_{j}\right)\left(a_{j}-b_{j}\right) \leq 0$. Therefore, from (2.27) we can obtain (2.25).

Similarly we can prove part (b).

\section{Results and discussion}

In the article, we establish a monotonicity property for the function involving the strongly convex function, prove the classical majorization theorem for majorized $n$-tuples by using strongly convex functions, give some applications of majorization theorem, provide a general inequality for a strongly convex function which gives the well-known inequalities such as Jensen's inequality and Slater's inequality for strongly convex functions. Furthermore, we also give some weighted versions of majorization theorem for certain $n$-tuples.

\section{Conclusion}

We find several majorization results for strongly convex functions and provide their applications. The given results improve the previous results. Our approach may have further applications in the theory of majorization. 


\section{Funding}

This work was supported by the Natural Science Foundation of China (Grant Nos. 61673169, 11701176, 11626101, 11601485), the Science and Technology Research Program of Zhejiang Educational Committee (Grant No. Y201635325)

\section{Competing interests}

The authors declare that they have no competing interests.

\section{Authors' contributions}

All authors contributed equally to the writing of this paper. All authors read and approved the final manuscript.

\section{Author details}

${ }^{1}$ Department of Mathematics, University of Peshawar, Peshawar, Pakistan. ${ }^{2}$ Department of Mathematics, Huzhou University, Huzhou, China.

\section{Publisher's Note}

Springer Nature remains neutral with regard to jurisdictional claims in published maps and institutional affiliations.

\section{Received: 18 September 2018 Accepted: 22 February 2019 Published online: 06 March 2019}

\section{References}

1. Eggleston, H.G.: Convexity. Cambridge University Press, New York (1958)

2. Asimow, L., Ellis, A.J.: Convexity Theory and Its Applications in Functional Analysis. Academic Press, New York (1980)

3. Zhang, X.-H., Wang, G.-D., Chu, Y.-M.: Convexity with respect to Hölder mean involving zero-balanced hypergeometric functions. J. Math. Anal. Appl. 353(1), 256-259 (2009)

4. Zhang, X.-M., Chu, Y.-M.: Convexity of the integral arithmetic mean of a convex function. Rocky Mt. J. Math. 40(3), 1061-1068 (2010)

5. Chu, Y.-M., Wang, M.-K., Jiang, Y.-P., Qiu, S.-L.: Monotonicity, convexity, and inequalities involving the Agard distortion function. Abstr. Appl. Anal. 2011, Article ID 671765 (2011)

6. Wang, M.-K., Chu, Y.-M., Qiu, S.-L., Jiang, Y.-P.: Convexity of the complete elliptic integrals of the first kind with respect to Hölder means. J. Math. Anal. Appl. 388(2), 1141-1146 (2012)

7. Barbu, V., Precupanu, T.: Convexity and Optimization in Banach Spaces. Springer, Dordrecht (2012)

8. Chu, Y.-M., Wang, M.-K.: Optimal Lehmer mean bounds for the Toader mean. Results Math. 61(3-4), 223-229 (2012)

9. Adil Khan, M., Khalid, S., Pečarić, J.: Refinements of some majorization type inequalities. J. Math. Inequal. 7(1), 73-92 (2013)

10. Yang, Z.-H., Qian, W.-M., Chu, Y.-M., Zhang, W.: Monotonicity rule for the quotient of two functions and its application. J. Inequal. Appl. 2017, Article ID 106 (2017)

11. Yang, Z.-H., Qian, W.-M., Chu, Y.-M., Zhang, W.: On rational bounds for the gamma function. J. Inequal. Appl. 2017, Article ID 210 (2017)

12. Qian, W.-M., Chu, Y.-M.: Sharp bounds for a special quasi-arithmetic mean in terms of arithmetic and geometric means with two parameters. J. Inequal. Appl. 2017, Article ID 274 (2017)

13. Zhao, T.-H., Wang, M.-K., Zhang, W., Chu, Y.-M.: Quadratic transformation inequalities for Gaussian hypergeometric function. J. Inequal. Appl. 2018, Article ID 251 (2018)

14. Yang, Z.-H., Qian, W.-M., Chu, Y.-M.: Monotonicity properties and bounds involving the complete elliptic integrals of the first kind. Math. Inequal. Appl. 21(4), 1185-1199 (2018)

15. Yang, Z.-H., Chu, Y.-M., Zhang, W.: High accuracy asymptotic bounds for the complete elliptic integral of the second kind. Appl. Math. Comput. 348, 552-564 (2019)

16. Wang, M.-K., Chu, Y.-M., Zhang, W.: The precise estimates for the solution of Ramanujan's generalized modular equation. Ramanujan J. https://doi.org/10.1007/s11139-018-0130-8

17. He, X.-H., Qian, W.-M., Xu, H.-Z., Chu, Y.-M.: Sharp power mean bounds for two Sándor-Yang means. Rev. R. Acad. Cienc. Exactas Fís. Nat., Ser. A Mat. https://doi.org/10.1007/s13398-019-00643-2

18. Qiu, S.-L., Ma, X.-Y., Chu, Y.-M.: Sharp Landen transformation inequalities for hypergeometric functions, with applications. J. Math. Anal. Appl. 474(2), 1306-1337 (2019)

19. de Finetti, B.: Sulle stratificazioni convesse. Ann. Mat. Pura Appl. (4) 30, 173-183 (1949)

20. Merentes, N., Nikodem, K.: Remarks on strongly convex functions. Aequ. Math. 80(1-2), 193-199 (2010)

21. Moradi, H.R., Omidvar, M.E., Adil Khan, M., Nikodem, K.: Around Jensen's inequality for strongly convex functions. Aequ. Math. 92(1), 25-37 (2018)

22. Hyers, D.H., Ulam, S.M.: Approximately convex functions. Proc. Am. Math. Soc. 3, 821-828 (1952)

23. Pečarić, J.E., Proschan, F., Tong, Y.L.: Convex Functions, Partial Orderings, and Statistical Applications. Academic Press, Boston (1992)

24. Jensen, J.L.W.V.: Om konvekse funktioner og uligheder imellem middelvaezrdier. Nyt Tidsskr. Math. 16, 49-69 (1905)

25. Mangasarian, O.L.: Pseudo-convex functions. J. Soc. Ind. Appl. Math., A, on Control 3, 281-290 (1965)

26. Varošanec, S.: On h-convexity. J. Math. Anal. Appl. 326(1), 303-311 (2007)

27. Rajba, T.: On strong delta-convexity and Hermite-Hadamard type inequalities for delta-convex functions of higher order. Math. Inequal. Appl. 18(1), 267-293 (2015)

28. Adil Khan, M., Chu, Y.-M., Khan, T.U., Khan, J.: Some new inequalities of Hermite-Hadamard type for s-convex functions with applications. Open Math. 15, 1414-1430 (2017)

29. Khurshid, Y., Adil Khan, M., Chu, Y.-M., Khan, Z.A., Liu, L.-S.: Hermite-Hadamard-Fejér inequalities for conformable fractional integrals via preinvex functions. J. Funct. Spaces 2019, Article ID 3146210 (2019)

30. Zhang, X.-M., Chu, Y.-M., Zhang, X.-H.: The Hermite-Hadamard type inequality of GA-convex functions and its applications. J. Inequal. Appl. 2010, Article ID 507560 (2010) 
31. Khurshid, Y., Adil Khan, M., Chu, Y.-M.: Conformable integral inequalities of the Hermite-Hadamard type in terms of GG- and GA-convexities. J. Funct. Spaces 2019, Article ID 6926107 (2019)

32. Adil Khan, M., Zaheer Ullah, S., Chu, Y.-M.: The concept of coordinate strongly functions and related inequalities. Rev. R. Acad. Cienc. Exactas Fís. Nat., Ser. A Mat. https://doi.org/10.1007/s13398-018-0615-8

33. Chu, Y.-M., LV, Y.-P.: The Schur harmonic convexity of the Hamy symmetric function and its applications. J. Inequal. Appl. 2009, Article ID 838529 (2009)

34. Chu, Y.-M., Xia, W.-F.: Solution of an open problem for Schur convexity or concavity of the Gini mean values. Sci. China 52A(10), 2099-2106 (2009)

35. Xia, W.-F., Chu, Y.-M.: Schur-convexity for a class of symmetric functions and its applications. J. Inequal. Appl. 2009, Article ID 493759 (2009)

36. Xia, W.-F., Chu, Y.-M.: Schur convexity and Schur multiplicative convexity for a class of symmetric functions with applications. Ukr. Math. J. 61(10), 1541-1555 (2009)

37. Xia, W.-F., Chu, Y.-M.: On Schur convexity of some symmetric functions. J. Inequal. Appl. 2010, Article ID 543250 (2010)

38. Chu, Y.-M., Xia, W.-F., Zhao, T.-H.: Schur convexity for a class of symmetric functions. Sci. China Math. 53(2), 465-474 (2010)

39. Chu, Y.-M., Wang, G.-D., Zhang, X.-H.: Schur convexity and Hadamard's inequality. Math. Inequal. Appl. 13(4), 725-731 (2010)

40. Chu, Y.-M., Sun, T.-C.: The Schur harmonic convexity for a class of symmetric functions. Acta Math. Sci. 30B(5), 1501-1506 (2010)

41. Chu, Y.-M., Wang, G.-D., Zhang, X.-H.: The Schur multiplicative and harmonic convexities of the complete symmetric function. Math. Nachr. 284(5-6), 653-663 (2011)

42. Xia, W.-F., Chu, Y.-M., Wang, G.-D.: Necessary and sufficient conditions for the Schur harmonic convexity or concavity of the extended mean values. Rev. Unión Mat. Argent. 52(1), 121-132 (2011)

43. Xia, W.-F., Chu, Y.-M.: The Schur convexity of Gini mean values in the sense of harmonic mean. Acta Math. Sci. 31B(3), $1103-1112(2011)$

44. Chu, Y.-M., Xia, W.-F., Zhang, X.-H.: The Schur concavity, Schur multiplicative and harmonic convexities of the second dual form of the Hamy symmetric function with applications. J. Multivar. Anal. 105, 412-421 (2012)

45. Yang, Z.-H., Qian, W.-M., Chu, Y.-M., Zhang, W.: On approximating the arithmetic-geometric mean and complete elliptic integral of the first kind. J. Math. Anal. Appl. 462(2), 1714-1726 (2018)

46. Yang, Z.-H., Zhang, W., Chu, Y.-M.: Sharp Gautschi inequality for parameter $0<p<1$ with applications. Math. Inequal. Appl. 20(4), 1107-1120 (2017)

47. Yang, Z.-H., Chu, Y.-M.: A monotonicity property involving the generalized elliptic integral of the first kind. Math. Inequal. Appl. 20(3), 729-735 (2017)

48. Wang, M.-K., Chu, Y.-M.: Refinements of transformation inequalities for zero-balanced hypergeometric functions. Acta Math. Sci. 37B(3), 607-622 (2017)

49. Qian, W.-M., Zhang, X.-H., Chu, Y.-M.: Sharp bounds for the Toader-Qi mean in terms of harmonic and geometric means. J. Math. Inequal. 11(1), 121-127 (2017)

50. Zhao, T.-H., Zhou, B.-C., Wang, M.-K., Chu, Y.-M.: On approximating the quasi-arithmetic mean. J. Inequal. Appl. 2019, Article ID 42 (2019)

51. Chu, Y.-M., Zhao, T.-H.: Concavity of the error function with respect to Hölder means. Math. Inequal. Appl. 19(2), 589-595 (2016)

52. Yang, Y.-Y., Qian, W.-M., Chu, Y.-M.: Refinements of bounds for Neumann means with applications. J. Nonlinear Sci. Appl. 9(4), 1529-1540 (2016)

53. Wang, M.-K., Chu, Y.-M., Song, Y.-Q.: Asymptotical formulas for Gaussian and generalized hypergeometric functions. Appl. Math. Comput. 276, 44-60 (2016)

54. Xia, W.-F., Chu, Y.-M.: Optimal inequalities for the convex combination of error function. J. Math. Inequal. 9(1), 85-99 (2015)

55. Yang, Z.-H., Chu, Y.-M., Wang, M.-K.: Monotonicity criterion for the quotient of power series with applications. J. Math. Anal. Appl. 428(1), 587-604 (2015)

56. Wang, G.-D., Zhang, X.-H., Chu, Y.-M.: A power mean inequality involving the complete elliptic integrals. Rocky Mt. J. Math. 44(5), 1661-1667 (2014)

57. Chu, Y.-M., Wang, M.-K., Ma, X.-Y.: Sharp bounds for Toader mean in terms of contraharmonic mean with applications. J. Math. Inequal. 7(2), 161-166 (2013)

58. Chu, Y.-M., Long, B.-Y.: Bounds of the Neuman-Sándor mean using power and identric means. Abstr. Appl. Anal. 2013 Article ID 832591 (2013)

59. Wang, M.-K., Chu, Y.-M.: Asymptotical bounds for complete elliptic integrals of the second kind. J. Math. Anal. Appl. 402(1), 119-126 (2013)

60. Chu, Y.-M., Qiu, Y.-F., Wang, M.-K.: Hölder mean inequalities for the complete elliptic integrals. Integral Transforms Spec. Funct. 23(7), 521-527 (2012)

61. Wang, M.-K., Chu, Y.-M., Qiu, S.-L., Jiang, Y.-P.: Bounds for the perimeter of an ellipse. J. Approx. Theory 164(7), 928-937 (2012)

62. Chu, Y.-M., Wang, M.-K., Qiu, S.-L., Jiang, Y.-P.: Bounds for complete elliptic integrals of the second kind with applications. Comput. Math. Appl. 63(7), 1177-1184 (2012)

63. Wang, M.-K., Wang, Z.-K., Chu, Y.-M.: An optimal double inequality between geometric and identric means. Appl. Math. Lett. 25(3), 471-475 (2012)

64. Wang, M.-K., Qiu, S.-L., Chu, Y.-M., Jiang, Y.-P.: Generalized Hersch-Pfluger distortion function and complete elliptic integrals. J. Math. Anal. Appl. 385(1), 221-229 (2012)

65. Wang, G.-D., Zhang, X.-H., Chu, Y.-M.: A power mean inequality for the Grötzsch ring function. Math. Inequal. Appl. 14(4), 833-837 (2011)

66. Wang, J.-L., Qian, W.-M., He, Z.-Y., Chu, Y.-M.: On approximating the Toader mean by other bivariate means. J. Funct. Spaces 2019, Article ID 6082413 (2019)

67. Chu, Y.-M., Adil Khan, M. Ali, T., Dragomir, S.S.: Inequalities for $\alpha$-fractional differentiable functions. J. Inequal. Appl. 2017, Article ID 93 (2017) 
68. Adil Khan, M., Wu, S.-H., Ullah, H., Chu, Y.-M.: Discrete majorization type inequalities for convex functions on rectangles. J. Inequal. Appl. 2019, Article ID 16 (2019)

69. Adil Khan, M., Khurshid, Y., Du, T.-S., Chu, Y.-M.: Generalization of Hermite-Hadamard type inequalities via conformable fractional integrals. J. Funct. Spaces 2018, Article ID 5357463 (2018)

70. Adil Khan, M., Iqbal, A., Suleman, M., Chu, Y.-M.: Hermite-Hadamard type inequalities for fractional integrals via Green's function. J. Inequal. Appl. 2018, Article ID 161 (2018)

71. Adil Khan, M., Chu, Y.-M., Kashuri, A., Liko, R., Ali, G.: Conformable fractional integrals versions of Hermite-Hadamard inequalities and their applications. J. Funct. Spaces 2018, Article ID 6928130 (2018)

72. Adil Khan, M., Begum, S., Khurshid, Y., Chu, Y.-M.: Ostrowski type inequalities involving conformable fractional integrals. J. Inequal. Appl. 2018, Article ID 70 (2018)

73. Huang, T.-R., Tan, S.-Y., Ma, X.-Y., Chu, Y.-M.: Monotonicity properties and bounds for the complete $p$-elliptic integrals. J. Inequal. Appl. 2018, Article ID 239 (2018)

74. Wang, M.-K., Qiu, S.-L., Chu, Y.-M.: Infinite series formula for Hübner upper bound function with applications to Hersch-Pfluger distortion function. Math. Inequal. Appl. 21(3), 629-648 (2018)

75. Xu, H.-Z., Chu, Y.-M., Qian, W.-M.: Sharp bounds for the Sándor-Yang means in terms of arithmetic and contra-harmonic means. J. Inequal. Appl. 2018, Article ID 127 (2018)

76. Huang, T.-R., Han, B.-W., Ma, X.-Y., Chu, Y.-M.: Optimal bounds for the generalized Euler-Mascheroni constant. J. Inequal. Appl. 2018, Article ID 118 (2018)

77. Wang, M.-K., Li, Y.-M., Chu, Y.-M.: Inequalities and infinite product formula for Ramanujan generalized modular equation function. Ramanujan J. 46(1), 189-200 (2018)

78. Wang, M.-K., Chu, Y.-M.: Landen inequalities for a class of hypergeometric functions with applications. Math. Inequal. Appl. 21(2), 521-537 (2018)

79. Yang, Z.-H., Qian, W.-M., Chu, Y.-M., Zhang, W.: On approximating the error function. Math. Inequal. Appl. 21(2), 469-479 (2018)

80. Marshall, A.W., Olkin, I.: Inequalities: Theory of Majorization and Its Applications. Academic Press, New York (1979)

81. Fuchs, L.: A new proof of an inequality of Hardy-Littlewood-Pólya. Mat. Tidsskr., B 1947, 53-54 (1947)

82. Niculescu, C.P., Persson, L.-E.: Convex Functions and Their Applications. Springer, New York (2006)

83. Dragomir, S.S.: Some majorisation type discrete inequalities for convex functions. Math. Inequal. Appl. 7(2), 207-216 (2004)

84. Kadelburg, Z., Dukić, D., Lukić, M., Matić, I.: Inequalities of Karamata, Schur and Muirhead, and some applications. Teach. Math. 8(1), 31-45 (2005)

85. Pečarić, J., Abramovich, S.: On new majorization theorems. Rocky Mt. J. Math. 27(3), 903-911 (1997)

\section{Submit your manuscript to a SpringerOpen ${ }^{\circ}$ journal and benefit from:}

- Convenient online submission

- Rigorous peer review

- Open access: articles freely available online

- High visibility within the field

- Retaining the copyright to your article

Submit your next manuscript at $\gg$ springeropen.com 